\title{
REVISÃO DA LITERATURA SOBRE EXTREMOS ANTROPOMÉTRICOS EM CRIANÇAS E ADOLESCENTES: PREVALÊNCIA, RISCOS À SAÚDE E FATORES SOCIODEMOGRÁFICOS ASSOCIADOS
}

\author{
LITERATURE REVIEW ON ANTHROPOMETRIC EXTREMES IN CHILDREN AND ADOLESCENTS: \\ PREVALENCE, HEALTH RISKS AND ASSOCIATED SOCIODEMOGRAPHIC FACTORS
}

\author{
Grazielle da Silva Souza ${ }^{a^{*}}$, Teresa Maria Bianchini de Quadros ${ }^{b^{*}}$, \\ Alex Pinheiro Gordia ${ }^{c^{*}}$, Vanessa Barbosa Facina ${ }^{\mathrm{d}^{*}}$ \\ agrazyhgt@gmail.com, btetemb@gmail.com, calexgordia@gmail.com, ‘vanessafacina@yahoo.com.br \\ *Universidade Federal do Recôncavo da Bahia - Amargosa (BA), Brasil
}

Data de recebimento do artigo: 06/12/2015 Data de aceite do artigo: 07/04/2015

\section{RESUMO}

Introdução: $\mathrm{O}$ Brasil vem passando por um processo de transição nutricional na infância e adolescência, marcado pelo aumento das prevalências de obesidade e declínio do baixo peso. Objetivo: Sistematizar achados referentes à prevalência, riscos à saúde e fatores sociodemográficos determinantes dos extremos antropométricos em crianças e adolescentes. Materiais e métodos: Foram pesquisados trabalhos em bases de dados de acordo com a seguinte estratégia de busca: "obesidade" OR "desnutriçáa" OR "adiposidade" OR "índice de massa corporal" OR "pregas cutâneas" AND "doenças cardiovasculares" OR "doença crônica" OR "fatores de risco" OR "grupos de risco" OR "mortalidade" OR "fatores socioeconômicos" OR "classe social" OR "condiçôes sociais", com filtro para a faixa etária pediátrica. Resultados: Foram analisados 39 artigos. No que se refere à obesidade/excesso de adiposidade, os resultados indicaram elevadas prevalências, variando de 13,3 a $40,7 \%$. A obesidade/excesso de adiposidade associou-se à hipertensão, hiperglicemia, dislipidemia e síndrome metabólica. Para o baixo peso/baixa reserva de adiposidade, observou-se variação de 2,1 a 14,9\% nas prevalências. Essa condiçáo mostrou-se associada à anemia ferropriva, hipertensão, doença falciforme, hipovitaminose A, problemas de saúde mental, déficit motor, déficit cognitivo e mortalidade precoce. Fatores sociodemográficos foram determinantes para os extremos antropométricos. Conclusóes: Observou-se coexistência entre elevada prevalência de obesidade/ excesso de adiposidade e prevalência ainda preocupante de baixo peso/baixa reserva de adiposidade em crianças e adolescentes. Houve forte associação entre extremos antropométricos e fatores de risco à saúde. Fatores sociodemográficos foram determinantes de ambas as condiçôes, e precisam ser considerados em programas que visem ao enfrentamento dos extremos antropométricos na infância e adolescência.

Palavras-chave: Obesidade; desnutrição; criança; adolescente; fatores de risco; fatores socioeconômicos.

\section{ABSTRACT}

Introduction: Brazil is facing a process of nutritional transition in childhood and adolescence, marked by an increase in prevalence of obesity and a reduction in underweight. Objective: To systematize findings on prevalence, health risks and determinants sociodemographic factors of extreme anthropometric measurements in children and adolescents. Materials and methods: Searched articles in databases according to the following search strategy: ("obesity" OR "malnutrition" OR "fat" OR "body mass index" OR "skinfold”) AND ("diseases cardiovascular" OR "chronic disease" OR "risk factors" OR "risk groups" OR "mortality" OR "socioeconomic factors" OR "social class" OR "social conditions"), with filter for the pediatric age group. Results: 39 articles were analyzed. With regard to obesity/excess body fat, the results indicated a high prevalence ranging from 13.3 to $40.7 \%$. Obesity/excess adiposity was associated with hypertension, hyperglycemia, dyslipidemia and metabolic syndrome. For low weight/low reserve adiposity, there was a growth of 2.1 to $14.9 \%$ in prevalence. This condition was associated with iron deficiency anemia, hypertension, sickle cell disease, vitamin A deficiency, mental health problems, motor deficits, cognitive impairment and early mortality. Sociodemographic factors were determinants for the extreme 
anthropometric measurements. Conclusions: There was a coexistence of high prevalence of obesity/excess body fat and worrying prevalence of underweight/low reserve adiposity among children and adolescents. There was a strong association between extreme anthropometric measurements and risk factors to health. Sociodemographic factors were determinants for both conditions and need to be considered in programs that cope with extreme anthropometric measurements in children and adolescents.

Keywords: Obesity; malnutrition; child; adolescent; risk factors; socioeconomic factors.

\section{Introdução}

Evidências indicam que o Brasil vem passando por um processo de transição nutricional entre crianças e adolescentes, marcada pelo aumento da prevalência de obesidade e declínio do baixo peso ${ }^{1-4}$. Entre 1974-1975 e 2008-2009 observou-se aumento de três a seis vezes na prevalência de obesidade entre crianças e adolescentes ${ }^{2}$. Por outro lado, a prevalência de baixo peso diminuiu, especialmente entre os adolescentes do sexo masculino ${ }^{2}$. Porém essa condição ainda representa um relevante problema de saúde entre jovens brasileiros, em especial na regiáo nordeste 2 .

Os extremos antropométricos estão associados a diversos fatores de risco à saúde durante a infância e adolescência ${ }^{3,5,6}$. Desde idades precoces, a obesidade/excesso de adiposidade aumenta a chance do desenvolvimento de doenças crônicas não transmissívei ${ }^{3-5}$, ao passo que o baixo peso/baixa reserva de adiposidade é um fator de predisposição para maior risco de doenças infecciosas e de mortalidade precoce ${ }^{6}$. Nesse sentido, os estudos que investigam os extremos antropométricos, bem como fatores associados, são relevantes para a elaboração de políticas públicas de promoção da saúde na infância e adolescência.

As causas da obesidade/excesso de adiposidade e do baixo peso/baixa reserva de adiposidade são multifatoriais e resultam da interação de fatores biológicos, sociais, comportamentais e culturais ${ }^{7}$. Nesse contexto, diversos estudos demonstraram associação entre os extremos antropométricos e fatores sociodemográficos ${ }^{8-17}$. Entre as variáveis associadas, pode-se citar a faixa etária ${ }^{8}$, o sexo ${ }^{8}$, o local de estudo ${ }^{9,10}$, o tipo de escola ${ }^{9,10}$, a escolaridade materna ${ }^{11,12}$, a renda familiar mensal $1^{8,13,14}$, a condição socioeconômica ${ }^{8,13,14}$, o número de irmãos ${ }^{15,16} \mathrm{e}$ o número de pessoas em casa ${ }^{17}$.

A quantidade de investigaçóes sobre os extremos antropométricos aumentaram significativamente nas últimas décadas. Há consenso de que essas condiçóes representam um preocupante problema de saúde pública. Contudo, a sistematização dos achados sobre o tema ainda é incipiente. Dessa forma, o presente estudo teve como objetivo realizar uma revisão da literatura referente à prevalência, riscos à saúde e fatores sociodemográficos determinantes aos extremos antropométricos, para subsidiar a atuação de profissionais e gestores de saúde no planejamento e desenvolvimento de programas de prevenção, proteção e promoção da saúde da população pediátrica.

\section{Métodos}

A revisão da literatura do presente estudo foi realizada nas bases de dados eletrônicas SciELO, Medline/ PubMed, LILACS e no site de busca Google Acadêmico por meio de pesquisa manual em periódicos brasileiros não indexados, busca específica por autores e em livros e documentos clássicos da área. Foram utilizados descritores, de forma isolada e combinada, em língua portuguesa e sua correspondência em inglês, de acordo com a seguinte estratégia de busca: "obesidade" OR "desnutrição" OR "adiposidade" OR "índice de massa corporal" OR "pregas cutâneas" AND "doenças cardiovasculares" OR "doença crônica" OR "fatores de risco" OR "grupos de risco" OR "mortalidade" OR "fatores socioeconômicos" OR "classe social” OR "condições sociais".

Utilizou-se filtro (nas bases de dados que possuíam esta ferramenta) para delimitar a faixa etária da busca na população pediátrica ( 0 a 19 anos). Estudos que não compreendiam esta faixa etária foram descartados. Não foi determinado um período de tempo específico de publicação na busca dos trabalhos. A seleção inicial dos artigos encontrados baseou-se na leitura do título, seguido da leitura do resumo. Apenas trabalhos que se adequaram ao tema "extremos antropométricos em crianças e adolescentes: prevalência, riscos à saúde e fatores sociodemográficos associados" foram incluídos para leitura na integra e análise detalhada. Apenas artigos originais foram incluídos na análise. Após a aplicaçâo desses critérios, 39 artigos originais foram analisados.

\section{Obesidade/excesso de adiposidade em crianças e adolescentes: prevalência e associação com fatores sociodemográficos}

A obesidade tornou-se um dos problemas de saúde que mais tem crescido nos últimos anos. Segundo a Organização Mundial da Saúde (OMS), desde 1980 os casos aumentaram em mais de $100 \%{ }^{18}$. No ano de 2010, cerca de 43 milhóes de crianças menores de cinco anos estavam acima do peso ${ }^{18}$. Há evidências de que $20 \%$ das crianças obesas antes de completar cinco anos de idade tendem a se tornar adultos obesos ${ }^{19}$. Quando a obesidade atinge o grupo dos adolescentes, a proporção 
pode aumentar para $80 \%{ }^{19}$. Diante dessa situação táo preocupante, reforça-se a necessidade de investigaçóes e açóes que visem mudar esse panorama.

No Quadro 1 podem ser observados os estudos que avaliaram a prevalência de obesidade/excesso de adiposidade e sua associação com variáveis sociodemográficas em crianças e adolescentes. Os achados dos estudos analisados indicaram elevada prevalência de obesidade/ excesso de adiposidade, confirmando que essa patologia representa um grave problema de saúde pública entre jovens. Nota-se que a maioria dos estudos analisados foram realizados nas regiōes Sul e Sudeste do Brasil, e as prevalências diferiram de uma regiáo para outra, destacando a necessidade de realizar-se mais estudos em outras regiōes do país.

$\mathrm{Na}$ maioria dos estudos avaliados, os meninos apresentaram maior prevalência da obesidade/excesso de adiposidade quando comparados às meninas. Os jovens pertencentes ao nível socioeconômico alto e médio apresentaram maior prevalência de obesidade/excesso de adiposidade. A zona de domicílio não foi um fator determinante de adiposidade periférica, ao passo que para a adiposidade central observou-se que escolares da zona rural apresentaram maior prevalência de excesso de adiposidade aos da zona urbana.

A probabilidade de apresentar obesidade/excesso de adiposidade foi maior em estudantes de escolas particulares. Ter mais irmãos ou mais pessoas em casa demonstrou ser um fator protetor para obesidade/excesso de adiposidade. Em alguns estudos, a escolaridade dos pais demonstrou ter relação com o estado nutricional das crianças e dos adolescentes.

O método mais utilizado nos estudos analisados para investigar o estado nutricional foi o índice de massa corporal (IMC). Contudo, há controvérsias sobre sua eficiência, uma vez que pode subestimar a gordura corporal e não diferenciá-la da massa muscular ${ }^{20}$. Nesse sentido, destaca-se a importância da realização de estudos que empreguem outros métodos para a investigação do estado nutricional na infância e adolescência. O método das dobras cutâneas (DC) tem se mostrado útil, pois grande proporção da gordura corporal se encontra no tecido subcutâneo, e, dessa forma, medidas quanto à sua espessura servem como indicador da quantidade de gordura corporal ${ }^{21}$. Misra et al. ${ }^{22}$ demonstraram que a DC subescapular (SE) tem melhor capacidade de prognosticar fatores de risco cardiovascular em adolescentes do que o IMC e o perímetro da cintura (PC). Não obstante, Montañés et al. ${ }^{23}$ evidenciaram que a DC triciptal (TR) foi um forte indicador de dislipidemias e hipertensão arterial em crianças e adolescentes.

É evidente o aumento significativo da prevalência de obesidade/excesso de adiposidade em crianças e adolescentes brasileiros $^{24}$. Dessa forma, identificar fatores sociodemográficos associados a esse desfecho é de extrema importância para prevenção de doenças e promoçáo da saúde, além de contribuir para o desenvolvimento de propostas de intervençóes, que devem incentivar a prática regular de atividade física e a adesão de hábitos alimentares mais saudáveis por parte da população pediátrica.

Quadro 1: Estudos sobre a prevalência de sobrepeso e obesidade e sua associação com variáveis sociodemográficas em crianças e adolescentes.

\begin{tabular}{|c|c|c|c|c|c|}
\hline Autor & Objetivo & $\begin{array}{c}\text { Faixa etária } \\
\text { (anos) }\end{array}$ & Variáveis & Principais achados & $\begin{array}{l}\text { Desfecho/ } \\
\text { prevalência }\end{array}$ \\
\hline Ronque et al. ${ }^{25}$ & $\begin{array}{l}\text { Verificar a prevalência de } \\
\text { sobrepeso e obesidade } \\
\text { em escolares de alto nível } \\
\text { socioeconômico. }\end{array}$ & 7 a 10 & IMC e DC & $\begin{array}{l}\text { Diferenças significativas entre os sexos } \\
\text { aos nove e dez anos, bem como no } \\
\text { conjunto de todas as idades }\end{array}$ & $\begin{array}{c}\text { Sobrepeso: } \\
\text { Meninos }=19,7 \% \\
\text { Meninas }=17,3 \% \\
\text { Obesidade: } \\
\text { Meninos }=17,5 \% \\
\text { Meninas }=9,3 \%\end{array}$ \\
\hline Duquia et al. ${ }^{8}$ & $\begin{array}{l}\text { Descrever e comparar as DC } \\
\text { (TR) e DC (SE) de acordo } \\
\text { com características demográ- } \\
\text { ficas, socioeconômicas, com- } \\
\text { portamentais e biológicas. }\end{array}$ & $\begin{array}{c}\text { Média de } \\
11,3\end{array}$ & $\mathrm{DC}$ & $\begin{array}{l}\text { O fator mais fortemente associado } \\
\text { com adiposidade nos meninos foi o ní- } \\
\text { vel socioeconômico e entre as meninas } \\
\text { foi o IMC materno. }\end{array}$ & $\begin{array}{c}\text { DC TR elevada: } \\
\text { Meninos }=20,2 \% \\
\text { Meninas }=14,2 \% \\
\text { DC SE elevada: } \\
\text { Meninos }=17,3 \% \\
\text { Meninas }=10,5 \%\end{array}$ \\
\hline Guedes et al. ${ }^{15}$ & $\begin{array}{l}\text { Analisar o impacto de fatores } \\
\text { sociodemográficos e com- } \\
\text { portamentais na prevalência } \\
\text { de sobrepeso e obesidade em } \\
\text { escolares. }\end{array}$ & 6 a 18 & IMC & $\begin{array}{l}\text { Classe econômica, escolaridade dos pais, } \\
\text { número de irmãos, tipo de alimentação } \\
\text { na escola, distância e meio de transporte } \\
\text { entre a moradia e a escola, realização de } \\
\text { trabalho remunerado e tipo de atividade } \\
\text { no lazer e no tempo livre. }\end{array}$ & $\begin{array}{c}\text { Sobrepeso: } \\
\text { Meninos }=14,7 \% \\
\text { Meninas }=19,7 \% \\
\text { Obesidade: } \\
\text { Meninos }=2,8 \% \\
\text { Meninas }=4,8 \%\end{array}$ \\
\hline
\end{tabular}

continua... 
Quadro 1: Continuação.

\begin{tabular}{|c|c|c|c|c|c|}
\hline Autor & Objetivo & $\begin{array}{c}\text { Faixa etária } \\
\text { (anos) }\end{array}$ & Variáveis & Principais achados & $\begin{array}{l}\text { Desfecho/ } \\
\text { prevalência }\end{array}$ \\
\hline Mello et al. ${ }^{16}$ & \begin{tabular}{|} 
Identificar a prevalência de \\
sobrepeso e obesidade e fatores \\
associados em alunos de seis a \\
dez anos das escolas públicas.
\end{tabular} & 6 a 10 & IMC & $\begin{array}{l}\text { Crianças com até um irmão apresenta- } \\
\text { ram maior probabilidade de apresentar } \\
\text { excesso de peso. }\end{array}$ & $\begin{array}{l}\frac{\text { Sobrepeso: }}{20,0 \%} \\
\text { Obesidade: } \\
7,0 \%\end{array}$ \\
\hline $\begin{array}{l}\text { Mendonça } \\
\text { et al. }{ }^{9}\end{array}$ & $\begin{array}{l}\text { Analisar a prevalência de } \\
\text { sobrepeso e obesidade em } \\
\text { crianças e adolescentes e veri- } \\
\text { ficar associaçáo com gênero, } \\
\text { faixa etária e tipo de escola. } \\
\end{array}$ & 7 a 17 & IMC & $\begin{array}{l}\text { Sobrepeso e obesidade foram signifi- } \\
\text { cantemente mais elevados em estudan- } \\
\text { tes das escolas particulares. }\end{array}$ & $\begin{array}{l}\frac{\text { Sobrepeso: }}{9,3 \%} \\
\frac{\text { Obesidade: }}{4,5 \%}\end{array}$ \\
\hline Minatto et al. ${ }^{14}$ & $\begin{array}{l}\text { Verificar a associação da com- } \\
\text { posição corporal inadequada } \\
\text { com fatores sociodemográfi- } \\
\text { cos em adolescentes. }\end{array}$ & 14 a 17 & $\begin{array}{c}\text { IMC, PC e } \\
\text { DC }\end{array}$ & $\begin{array}{l}\text { Adolescentes do sexo masculino e dos } \\
\text { estratos econômicos alto e intermediá- } \\
\text { rio tiveram maiores índices inadequa- } \\
\text { dos de adiposidade. }\end{array}$ & $\begin{array}{c}\frac{\text { Composiçáo cor- }}{\text { poral inadequada: }} \\
24,1 \%\end{array}$ \\
\hline Ferrari et al. ${ }^{26}$ & $\begin{array}{l}\text { Analisar as modificaçóes } \\
\text { da adiposidade de escolares } \\
\text { durante } 20 \text { anos. }\end{array}$ & 7 a 10 & IMC e DC & $\begin{array}{l}\text { Meninos e meninas aumentaram sua } \\
\text { adiposidade no grupo com excesso de } \\
\text { peso. }\end{array}$ & $\begin{array}{c}\text { Sobrepeso: } \\
\text { Meninos }=18,3 \% \\
\text { Meninas }=26,9 \% \\
\text { Obesidade: } \\
\text { Meninos }=16,9 \% \\
\text { Meninas }=14,7 \%\end{array}$ \\
\hline Leal et al. ${ }^{17}$ & $\begin{array}{l}\text { Verificar a prevalência e os } \\
\text { determinantes do excesso } \\
\text { ponderal em crianças e } \\
\text { adolescentes. }\end{array}$ & 5 a 19 & IMC & $\begin{array}{l}\text { Maior renda familiar e escolaridade } \\
\text { materna, posse de bens de consumo, } \\
\text { residência em área urbana e o excesso } \\
\text { de peso materno foram determinantes } \\
\text { para o excesso de peso dos jovens. }\end{array}$ & $\begin{array}{l}\frac{\text { Sobrepeso: }}{9,5 \%} \\
\text { Obesidade: } \\
3,8 \%\end{array}$ \\
\hline Quadros et al. ${ }^{27}$ & $\begin{array}{c}\text { Avaliar o desempenho dos } \\
\text { pontos de corte para o IMC } \\
\text { recomendados por Cole et } \\
\text { al. e Conde e Monteiro para } \\
\text { diagnosticar o excesso de peso } \\
\text { em crianças. }\end{array}$ & 6 a 9 & IMC e DC & $\begin{array}{l}\text { O critério de Conde e Monteiro } \\
\text { mostrou-se mais sensível, acarretando } \\
\text { menor número de falso-negativos. }\end{array}$ & $\begin{array}{c}\text { Excesso de peso: } \\
20,7 \% \\
\text { (Cole et al.) } \\
28,9 \% \\
\text { (Conde e } \\
\text { Monteiro) } \\
\end{array}$ \\
\hline Cesani et al. ${ }^{28}$ & $\begin{array}{l}\text { Analisar a relação do estado } \\
\text { nutricional e composição } \\
\text { corporal com o ambiente de } \\
\text { residência (urbana ou rural) } \\
\text { de crianças. }\end{array}$ & 3 a 14 & $\begin{array}{l}\text { IMC, DC } \\
\text { e PB }\end{array}$ & $\begin{array}{c}\text { O ambiente de residência não promo- } \\
\text { veu qualquer diferenciaçáo no estado } \\
\text { nutricional. No entanto, o incremento } \\
\text { de adiposidade central e, em alguns } \\
\text { casos, de déficit muscular em crianças } \\
\text { da zona rural, sugeriu um consumo } \\
\text { desequilibrado na dieta. }\end{array}$ & $\begin{array}{l}\frac{\text { Sobrepeso: }}{12,1 \%} \\
\text { Obesidade: } \\
9,7 \%\end{array}$ \\
\hline Benedet et al. ${ }^{10}$ & $\begin{array}{c}\text { Estimar a prevalência de } \\
\text { excesso de peso e identifi- } \\
\text { car associaçóes com fatores } \\
\text { sociodemográficos, bioló- } \\
\text { gicos e de estilo de vida em } \\
\text { adolescentes. }\end{array}$ & 11 a 14 & IMC & $\begin{array}{c}\text { Em meninos, o excesso de peso asso- } \\
\text { ciou-se com a não realizaçáo de refeições } \\
\text { estruturadas e com o deslocamento } \\
\text { não ativo para a escola. Em meninas, o } \\
\text { excesso de peso materno, a não realização } \\
\text { de refeições estruturadas e a maturação } \\
\text { sexual se associaram com excesso de peso. }\end{array}$ & $\begin{array}{c}\text { Excesso de peso: } \\
19,3 \% \\
\text { (Cole et al.) } \\
25,8 \% \\
\text { (Conde e } \\
\text { Monteiro) }\end{array}$ \\
\hline $\begin{array}{c}\text { Vázquez-Nava } \\
\text { et al. }{ }^{29}\end{array}$ & $\begin{array}{l}\text { Determinar a associação entre } \\
\text { a estrutura familiar, o nível de } \\
\text { escolaridade e emprego da mãe } \\
\text { com o estilo de vida sedentário } \\
\text { e estado nutricional de crianças. }\end{array}$ & 6 a 12 & IMC & $\begin{array}{l}\text { Morar com uma família de pais separa- } \\
\text { dos, ter um baixo nível de escolaridade } \\
\text { materno e ter mãe que trabalha fora } \\
\text { parecem estar associados ao sobrepeso. }\end{array}$ & $\frac{\text { Excesso de peso: }}{40,7 \%}$ \\
\hline Minatto et al..$^{30}$ & $\begin{array}{l}\text { Estimar a prevalência de adi- } \\
\text { posidade corporal elevada e } \\
\text { sua associaçáo com a aptidáo } \\
\text { musculoesquelética por nível } \\
\text { econômico em crianças e ado- } \\
\text { lescentes do sexo masculino. }\end{array}$ & 6 a 17 & DC & $\begin{array}{l}\text { A prevalência de adiposidade corporal } \\
\text { elevada foi maior em adolescentes do } \\
\text { nível econômico alto. }\end{array}$ & $\begin{array}{l}\frac{\text { Adiposidade }}{\text { corporal elevada: }} \\
30,4 \%\end{array}$ \\
\hline
\end{tabular}

IMC: índice de massa corporal; DC: dobra cutânea; PC: perímetro da cintura, PB: perímetro do braço. 


\section{Obesidade/excesso de adiposidade: associação com fatores de risco à saúde em crianças e adolescentes}

A obesidade é uma doença crônica, com consequências graves para a saúde. $\mathrm{O}$ desenvolvimento precoce de fatores de risco cardiovascular, como a hipertensão arterial, a hiperglicemia e elevados níveis de lipoproteínas de baixa densidade estão associados aos elevados níveis de adiposidade ${ }^{31}$. Ferrari et al. ${ }^{26}$ demonstraram que o acúmulo de gordura subcutânea central tem aumentado de forma mais acentuada do que a adiposidade total avaliada a partir do IMC, ou seja, mesmo em populações sem alteração no IMC podem ter ocorrido mudanças desfavoráveis na composiçáo e distribuição da gordura corporal para um perfil associado a maiores riscos de doenças ${ }^{26}$.

Os estudos sobre a associação da obesidade/excesso de adiposidade com fatores de risco à saúde em crianças e adolescentes podem ser verificados no Quadro 2. Os achados indicaram a importância de um diagnóstico precoce do estado nutricional, já que a obesidade/excesso de adiposidade vêm demonstrando associação positiva com doenças crônicas não transmissíveis. A maioria dos estudos encontrados analisou a associaçáo da obesidade/excesso de adiposidade com os valores pressóricos, e indicou associação robusta entre a hipertensão arterial e a obesidade/excesso de adiposidade. Além disso, os escolares com obesidade/excesso de adiposidade apresentaram maior probabilidade de apresentar colesterol total elevado. Contudo, variáveis como dislipidemias, hiperglicemia e síndrome metabólica foram pouco investigadas. Foi observado ainda que há um reduzido número de pesquisas que analisam a associação das DC com fatores de risco à saúde, deixando evidente a necessidade de estudos nesse sentido.

Apesar da importância do diagnóstico dos casos de sobrepeso e obesidade nas fases iniciais da vida, os Parâmetros Curriculares Nacionais da disciplina de Educação Física ${ }^{32}$ não mencionam a importância do emprego da avaliação antropométrica na escola. Guedes ${ }^{33}$ sugere que a escola, e especialmente a educação física, atuem diretamente com programas de açóes favoráveis para o controle e/ou prevenção da obesidade/excesso de adiposidade.

Mazzoccante et al. $^{34}$ realizaram uma pesquisa na qual a maioria dos professores de educação física não realizam avaliaçóes antropométricas em seus alunos, e dentre os que a realizam, poucos retornam os resultados aos alunos, pais e/ou responsáveis, especialmente em escolas públicas. Alguns professores justificaram não receber apoio da equipe pedagógica da escola, o que pode dificultar a realização do trabalho. De acordo com os autores, a participação efetiva de toda a comunidade escolar é uma importante ferramenta no diagnóstico do estado nutricional dos alunos, permitindo uma influência positiva na prevenção da obesidade/excesso de adiposidade tanto nas aulas quanto em atividades promovidas pela escola ${ }^{34}$.

Com base em nossos resultados, destaca-se a relevância do diagnóstico cada vez mais precoce dos casos de obesidade/excesso de adiposidade, já que vêm demonstrando associação positiva com fatores de risco à saúde. Medidas de intervenção para o desenvolvimento de um estilo de vida mais saudável devem ter início na infância e na adolescência, com a finalidade de diminuir a incidência de doenças crônicas não transmissíveis na idade adulta.

Quadro 2: Estudos sobre a associação da obesidade/excesso de adiposidade com fatores de risco à saúde em crianças e adolescentes.

\begin{tabular}{|c|c|c|c|c|c|}
\hline Autor & Objetivo & $\begin{array}{c}\text { Faixa etária } \\
\text { (anos) }\end{array}$ & $\begin{array}{c}\text { Variáveis } \\
\text { antropométricas }\end{array}$ & $\begin{array}{l}\text { Desfechos } \\
\text { analisados }\end{array}$ & Principais achados \\
\hline $\begin{array}{l}\text { Ribeiro } \\
\text { et al. } .^{5}\end{array}$ & $\begin{array}{c}\text { Examinar a associaçáo } \\
\text { de sobrepeso e obesidade } \\
\text { com perfis de atividade } \\
\text { física, PA e lípides séricos. }\end{array}$ & 6 a 18 & IMC, DC, PC e PP & $\begin{array}{c}\text { CT, LDL, HDL, } \\
\text { PAS e PAD }\end{array}$ & $\begin{array}{l}\text { Escolares com sobrepeso, obesos ou nos } \\
\text { quartis superiores para outras variáveis } \\
\text { de adiposidade apresentaram maiores } \\
\text { valores para PA e perfil lipídico de risco. }\end{array}$ \\
\hline $\begin{array}{l}\text { Freedman } \\
\text { et al. }{ }^{35}\end{array}$ & $\begin{array}{l}\text { Explorar a associação do } \\
\text { IMC com a adiposida- } \\
\text { de corporal, lipídios, } \\
\text { insulina e PA. }\end{array}$ & Média de 11,4 & IMC e DC & $\begin{array}{l}\text { Perfil lipídico, } \\
\text { insulina e PA }\end{array}$ & $\begin{array}{l}\text { Dentre as crianças com IMC acima do } \\
\text { percentil } 95,39 \% \text { tiveram pelo menos } \\
\text { dois fatores de risco, } 65 \% \text { tinham exces- } \\
\text { so de adiposidade. } \\
\text { Dentre as crianças com IMC acima do } \\
\text { percentil } 99,59 \% \text { tinham pelo menos } \\
\text { dois fatores de risco e } 94 \% \text { tinham } \\
\text { excesso de adiposidade. }\end{array}$ \\
\hline $\begin{array}{l}\text { Ferreira e } \\
\text { Aydos }^{36}\end{array}$ & $\begin{array}{l}\text { Investigar a relação entre } \\
\text { a HA e adiposidade } \\
\text { corporal em crianças e } \\
\text { adolescentes obesos. }\end{array}$ & 6 a 14 & IMC,\%GC e RCQ & PA & $\begin{array}{l}\text { O grupo dos hipertensos apresentou } \\
\text { níveis mais elevados de IMC e de \%GC } \\
\text { comparado ao grupo dos normotensos. }\end{array}$ \\
\hline
\end{tabular}

continua.. 
Quadro 2: Continuação.

\begin{tabular}{|c|c|c|c|c|c|}
\hline Autor & Objetivo & $\begin{array}{c}\text { Faixa etária } \\
\text { (anos) }\end{array}$ & $\begin{array}{c}\text { Variáveis } \\
\text { antropométricas }\end{array}$ & $\begin{array}{l}\text { Desfechos } \\
\text { analisados }\end{array}$ & Principais achados \\
\hline $\begin{array}{l}\text { Rinaldi et } \\
\mathrm{al}^{3}{ }^{3}\end{array}$ & $\begin{array}{l}\text { Verificar a prevalência } \\
\text { de HA e sua associa- } \\
\text { ção com indicadores } \\
\text { antropométricos. }\end{array}$ & 6 a 14 & IMC, PB, PC e DC & PA & $\begin{array}{l}\text { Houve correlação positiva e significa- } \\
\text { tiva entre os níveis de PAS e PAD e as } \\
\text { variáveis antropométricas. }\end{array}$ \\
\hline $\begin{array}{c}\text { Martins et } \\
\mathrm{al}^{37}\end{array}$ & $\begin{array}{c}\text { Investigar a associação } \\
\text { do sobrepeso e obesida- } \\
\text { de com a HA. }\end{array}$ & 12 a 18 & IMC & PA e PH & $\begin{array}{l}\text { Somente a obesidade esteve associada à } \\
\text { PA elevada entre os meninos. Em me- } \\
\text { ninas, sobrepeso e obesidade estiveram } \\
\text { associados à PA elevada. }\end{array}$ \\
\hline $\begin{array}{l}\text { Reuter et } \\
\text { al. }^{38}\end{array}$ & $\begin{array}{l}\text { Avaliar a relação entre } \\
\text { obesidade e fatores de } \\
\text { risco cardiovascular. }\end{array}$ & 8 a 17 & IMC e PC & $\begin{array}{l}\text { PAS, PAD } \\
\text { CT, glicemia e } \\
\text { triglicerídeos }\end{array}$ & $\begin{array}{l}\text { Os escolares com sobrepeso/obesidade } \\
\text { apresentaram percentual superior para } \\
\text { os indicadores bioquímicos e pressóri- } \\
\text { cos em comparação aos escolares com } \\
\text { baixo peso/peso normal. }\end{array}$ \\
\hline Silva et al. ${ }^{39}$ & $\begin{array}{c}\text { Influência de fatores } \\
\text { antropométricos e } \\
\text { atividade física na PA de } \\
\text { adolescentes. }\end{array}$ & 11 a 13 & IMC & PA & $\begin{array}{l}\text { Sobrepeso e obesidade estiveram asso- } \\
\text { ciados ao desenvolvimento de hiperten- } \\
\text { são arterial. }\end{array}$ \\
\hline $\begin{array}{l}\text { Sousa et } \\
\text { al. }^{40}\end{array}$ & $\begin{array}{c}\text { Estudar a associação } \\
\text { entre PN e fatores de } \\
\text { risco cardiovascular em } \\
\text { adolescentes. }\end{array}$ & 11 a 18 & $\mathrm{PN}$ & $\begin{array}{l}\text { PC, PA, lipídios, } \\
\text { glicemia, insuli- } \\
\text { na, resistência in- } \\
\text { sulínica e síndro- } \\
\text { me metabólica }\end{array}$ & $\begin{array}{l}\text { Observou-se maior frequência de obe- } \\
\text { sidade, PAS e PAD elevadas e síndrome } \\
\text { metabólica no grupo com PN alto em } \\
\text { relação ao grupo com PN normal. }\end{array}$ \\
\hline
\end{tabular}

IMC: Índice de massa corporal; DC: Dobra cutânea; PC: Perímetro da cintura; PP: Perímetro pélvico; PB: Perímetro braquial; RCQ: Razão cintura quadril; \%GC: Percentual de gordura corporal; PA: Pressão arterial; PAS: Pressão arterial sistólica; PAD: Pressão arterial diastólica; HA: Hipertensão arterial; PH: Préhipertensão; CT: Colesterol total; LDL: Lipoproteínas de baixa densidade; HDL: Lipoproteínas de alta densidade; PN: Peso ao nascer.

\section{Baixo peso/baixa reserva de adiposidade: prevalência e associação com fatores sociodemográficos em crianças e adolescentes}

A desnutrição é uma condição patológica decorrente da falta de energia e proteínas em variadas proporções, podendo ser agravada por infecçóes repetidas ${ }^{41}$. As crianças, por serem mais vulneráveis a deficiências nutricionais, constituem o grupo indicador preferencial para o estudo da presença de desnutriçáo em uma população, admitindo-se que a proporção de crianças com baixa estatura, de acordo com o padrão internacional de crescimento recomendado pela $\mathrm{OMS}^{42}$, pode retratar não apenas a prevalência da desnutriçáo na infância, mas indicar a dimensão global que o problema da desnutrição alcança na sociedade ${ }^{43}$.

Dados da Pesquisa de Orçamentos Familiares (POF) indicaram que houve uma queda significativa na desnutriçáo em crianças de cinco a nove anos no Brasil. Para os meninos, a prevalência foi de 29,3\% em 1974-1975 para 7,2\% em 2008-2009 ${ }^{2}$. Entre as meninas, o índice caiu de $26,7 \%$ para $6,3 \%$ neste mesmo período. Entre os adolescentes de 10 a 19 anos houve um declínio de $10,1 \%$, sendo $3,7 \%$ entre os rapazes e de $5,1 \%$ para $3,0 \%$ entre as moças ${ }^{2}$. Contudo, apesar da prevalência da desnutrição e/ou do baixo peso/baixa reserva de adiposidade ter diminuído ao longo das últimas décadas, de acordo com a literatura essa condição ainda é presente em várias regióes do Brasil, e pode estar ligada a fatores ambientais e socioeconômicos, como escolaridade paterna e materna, número de irmãos, estado empregatício do chefe da família, tipo de moradia, número de pessoas em casa, tipo de disposição do esgoto, acesso à água encanada e tratada e renda per capita ${ }^{44}$.

No Quadro 3 pode-se observar os estudos que verificaram a prevalência de baixo peso/baixa reserva de adiposidade e sua associação com variáveis sociodemográficas. Podemos ressaltar que, de acordo com os estudos analisados, mesmo com a elevada prevalência de obesidade/excesso de adiposidade observada nos últimos anos, o baixo peso/baixa reserva de adiposidade ainda atinge uma parcela considerável de crianças e adolescentes. Resultados da POF revelaram que as prevalências de baixo peso diferem entre as regióes do Brasil, sendo que as regióes Norte e Nordeste apresentam maiores prevalências ${ }^{2}$. Acredita-se que o baixo desenvolvimento econômico dessas regiōes pode ser uma das justificativas para tal achado.

Nos estudos analisados, pôde-se observar que os meninos apresentaram maior prevalência de baixo peso/ 
baixa reserva de adiposidade quando comparados às meninas. A escolaridade materna parece afetar o estado nutricional das crianças e dos adolescentes, pois os filhos cujas máes tinham o menor nível de escolaridade apresentaram mais probabilidade de apresentar baixo peso/baixa reserva de adiposidade. Além disso, de acordo com a presente revisão, dividir a casa com duas pessoas ou mais por cômodo parece afetar o estado nutricional, pois quanto maior o número de pessoas na casa, maiores são as chances de baixo peso/baixa reserva de adiposidade, além de fatores como tipo de residência, número de cômodos na casa, origem da água e peso ao nascer. No decorrer desta revisão de literatura pôde-se notar a escassez de estudos atuais sobre essa temática, destacando a importância de novos estudos, para identificar quais são os grupos de risco para o baixo peso/ baixa reserva de adiposidade, com o objetivo de realizar medidas de tratamento e prevenção.

Quadro 3: Estudos sobre a prevalência de baixo peso/baixa reserva de adiposidade e sua associação com variáveis sociodemográficas.

\begin{tabular}{|c|c|c|c|c|c|}
\hline Autor & Objetivo & $\begin{array}{c}\text { Faixa etária } \\
\text { (anos) }\end{array}$ & Variáveis & Principais achados & Desfecho/prevalência \\
\hline $\begin{array}{l}\text { Pelegrini et } \\
\text { al. }{ }^{45}\end{array}$ & $\begin{array}{l}\text { Verificar o estado nutricional de } \\
\text { escolares e analisar sua associa- } \\
\text { çáo com fatores demográficos e } \\
\text { nível de atividade física. }\end{array}$ & 10 a 17 & $\begin{array}{c}\text { IMC, sexo, idade, } \\
\text { área de domicílio e } \\
\text { nível de atividade } \\
\text { física }\end{array}$ & $\begin{array}{c}\text { As moças de } 10 \text { a } 13 \text { anos } \\
\text { apresentaram maior chance } \\
\text { de desnutriçáa. }\end{array}$ & $\begin{array}{c}\text { Baixo peso } \\
11,4 \%\end{array}$ \\
\hline Silveira et al. ${ }^{46}$ & $\begin{array}{l}\text { Investigar a associação da des- } \\
\text { nutrição em crianças residentes } \\
\text { em assentamentos subnormais } \\
\text { (favelas) com o estado nutri- } \\
\text { cional materno e as condiçóes } \\
\text { socioambientais. }\end{array}$ & 0 a 6 & $\begin{array}{l}\text { Peso, estatura, } \\
\text { idade e sexo }\end{array}$ & $\begin{array}{c}\text { A desnutrição crônica asso- } \\
\text { ciou-se à idade, escolaridade } \\
\text { materna, tipo de residência, } \\
\text { número de cômodos, reves- } \\
\text { timento de piso, origem da } \\
\text { água e PN. } \\
\end{array}$ & $\begin{array}{c}\text { Desnutrição crônica } \\
8,6 \%\end{array}$ \\
\hline $\begin{array}{l}\text { Ferreira; } \\
\text { Luciano }^{47}\end{array}$ & $\begin{array}{l}\text { Estimar a prevalência de extre- } \\
\text { mos antropométricos indicati- } \\
\text { vos do estado nutricional }\end{array}$ & 0 a 5 & $\begin{array}{l}\text { Peso, estatura, } \\
\text { idade, sexo e área } \\
\text { de moradia }\end{array}$ & $\begin{array}{c}\text { O déficit de estatura acome- } \\
\text { teu com maior intensidade } \\
\text { crianças com idade entre } 24 \\
\text { e } 36 \text { meses. A prevalência } \\
\text { de desnutrição foi maior } \\
\text { entre as crianças das áreas } \\
\text { rurais. }\end{array}$ & $\begin{array}{l}\text { Baixo peso } \\
2,9 \% \\
\text { Magreza } \\
1,2 \% \\
\text { Nanismo } \\
10,4 \%\end{array}$ \\
\hline Krinski et al. ${ }^{48}$ & $\begin{array}{c}\text { Analisar o estado nutricional e } \\
\text { verificar sua associaçáo com o } \\
\text { gênero e a idade }\end{array}$ & 6 a 17 & IMC, idade e sexo & $\begin{array}{c}\text { Meninas apresentaram pre- } \\
\text { valência de baixo peso mais } \\
\text { elevada do que meninos. }\end{array}$ & $\begin{array}{c}\text { Baixo peso } \\
\text { Meninos }=2,0 \% \\
\text { Meninas }=5,2 \%\end{array}$ \\
\hline Barros et al. ${ }^{49}$ & $\begin{array}{l}\text { Avaliar a composição nutri- } \\
\text { cional e a aceitabilidade da } \\
\text { alimentação escolar, o estado } \\
\text { nutricional e a segurança ali- } \\
\text { mentar de escolares. }\end{array}$ & 10 a 19 & $\begin{array}{l}\text { IMC, estatura, } \\
\text { idade e sexo }\end{array}$ & $\begin{array}{c}\text { Somente dois alunos apre- } \\
\text { sentaram baixo peso, sendo } \\
\text { um de cada sexo, e somente } \\
\text { um aluno apresentou déficit } \\
\text { de estatura para idade. }\end{array}$ & $\begin{array}{c}\text { Baixo peso } \\
1,7 \% \\
\text { Baixa estatura para } \\
\text { idade } \\
0,8 \% \\
\end{array}$ \\
\hline Benício et al. ${ }^{50}$ & $\begin{array}{c}\text { Estimar a prevalência da desnu- } \\
\text { triçáo infantil para os municí- } \\
\text { pios brasileiros. }\end{array}$ & 0 a 5 & $\begin{array}{l}\text { Estatura, sexo, } \\
\text { idade, regiōes } \\
\text { brasileiras, sexo, } \\
\text { número de pessoas } \\
\text { no domicílio, } \\
\text { nível econômico }\end{array}$ & $\begin{array}{l}\text { O risco de desnutrição foi } \\
\text { maior em crianças do sexo } \\
\text { masculino que moravam } \\
\text { em domicílios com duas ou } \\
\text { mais pessoas por cômodo, } \\
\text { pertencentes aos quin- } \\
\text { tos inferiores do escore } \\
\text { socioeconômico; com três } \\
\text { ou mais crianças < } 5 \text { anos } \\
\text { que não dispunham de água } \\
\text { encanada ou que residiam } \\
\text { na região Norte do Brasil. }\end{array}$ & $\begin{array}{c}\text { Desnutrição } \\
\text { Variando de } 2,8 \% \text { a } \\
\text { 29,0\% a depender do } \\
\text { município estudado }\end{array}$ \\
\hline Flores et al. ${ }^{4}$ & $\begin{array}{c}\text { Descrever e analisar a tendên- } \\
\text { cia da ocorrência do baixo } \\
\text { peso, sobrepeso e obesidade de } \\
\text { escolares. }\end{array}$ & 7 a 14 & IMC, sexo e idade & $\begin{array}{c}\text { A probabilidade de baixo } \\
\text { peso é maior em crianças } \\
\text { e adolescentes do sexo } \\
\text { feminino. }\end{array}$ & $\begin{array}{l}\text { Baixo peso } \\
2,1 \%\end{array}$ \\
\hline
\end{tabular}


Quadro 3: Continuação.

\begin{tabular}{|c|c|c|c|c|c|}
\hline Autor & Objetivo & $\begin{array}{c}\text { Faixa etária } \\
\text { (anos) }\end{array}$ & Variáveis & Principais achados & Desfecho/prevalência \\
\hline Guedes et al..$^{51}$ & $\begin{array}{l}\text { Analisar a prevalência de baixo } \\
\text { peso corporal/magreza, sobre- } \\
\text { peso e obesidade em crianças } \\
\text { e adolescentes de uma região } \\
\text { brasileira de baixo desenvolvi- } \\
\text { mento econômico. }\end{array}$ & 7 a 17 & IMC, sexo e idade & $\begin{array}{c}\text { A prevalência de baixo peso } \\
\text { foi maior em adolescentes } \\
\text { e em indivíduos do sexo } \\
\text { masculino. }\end{array}$ & $\begin{array}{c}\text { Baixo peso } \\
\text { Meninos }=6,3 \% \\
\text { Meninas }=4,1 \%\end{array}$ \\
\hline Pinho et al..$^{2}$ & $\begin{array}{l}\text { Identificar a prevalência do } \\
\text { excesso de peso e o consumo } \\
\text { alimentar entre adolescentes da } \\
\text { rede pública de ensino. }\end{array}$ & 11 a 17 & IMC & $\begin{array}{l}\text { Meninos apresentaram } \\
\text { maior proporção de baixo } \\
\text { peso. }\end{array}$ & $\begin{array}{c}\text { Baixo peso } \\
\text { Meninos }=11,1 \% \\
\text { Meninas }=3,8 \%\end{array}$ \\
\hline Correia et al..$^{53}$ & $\begin{array}{l}\text { Analisar tendências na preva- } \\
\text { lência e fatores determinantes } \\
\text { da desnutrição em crianças na } \\
\text { região semiárida do Brasil. }\end{array}$ & 0 a 3 & $\begin{array}{c}\text { Peso e altura/ } \\
\text { comprimento para } \\
\text { idade; peso para } \\
\text { altura }\end{array}$ & $\begin{array}{l}\text { Em } 1987 \text {, as características } \\
\text { socioeconômicas e biológi- } \\
\text { cas foram fatores signifi- } \\
\text { cativamente associados à } \\
\text { desnutrição, ao nanismo e } \\
\text { à emaciação. Em 2007, os } \\
\text { determinantes da desnu- } \\
\text { trição ficaram restritos às } \\
\text { características biológicas. }\end{array}$ & $\begin{array}{c}\text { Desnutrição aguda } \\
\text { 12,6\% em } 1987 \\
\text { 4,7\% em } 2007 \\
\text { Nanismo } \\
\text { 27,0\% em } 1987 \\
\text { 13,0\% em } 2007\end{array}$ \\
\hline
\end{tabular}

IMC: Índice de massa corporal; PN: Peso ao nascer.

\section{Baixo peso/baixa reserva de adiposidade: associação com fatores de risco à saúde em crianças e adolescentes}

A desnutrição pode ser definida como uma condição clínica decorrente de uma deficiência ou excesso, relativo ou absoluto, de um ou mais nutrientes essenciais. Pode ser considerada um estado de carência nutricional, um distúrbio com desaceleração (casos leves), interrupção (casos moderados), ou involução (casos graves) do processo de crescimento e desenvolvimento. Nas crianças e adolescentes, a desnutrição pode trazer prejuízos bioquímicos, funcionais e anatômicos que podem ou não ser reversíveis, chegando a ser fatai ${ }^{54}$.

A desnutrição é responsável por $55 \%$ das mortes de crianças no mundo inteiro e está associada a várias outras doenças, sendo ainda hoje considerada a doença que mais mata crianças abaixo de cinco anos ${ }^{55}$. No Quadro 4 estão sumarizados estudos que investigaram a prevalência de baixo peso/baixa reserva de adiposidade e sua associação com fatores de risco à saúde em crianças e adolescentes. O diagnóstico precoce do baixo peso/ baixa reserva de adiposidade é de extrema importância, pois esse desfecho pode acarretar prejuízos à saúde do indivíduo, muitas vezes irreversíveis.

Segundo a $\mathrm{OMS}^{42}$, o baixo peso ao nascer refere-se a crianças com peso inferior a $2,5 \mathrm{~kg}$ no momento do nascimento. Os estudos avaliados na presente revisão demonstraram que a desnutrição infanto-juvenil está diretamente ligada ao baixo peso ao nascer, destacando a relevância do acompanhamento gestacional, tendo em vista que as distribuiçóes do peso ao nascer e as condiçóes de saúde são determinadas por diversos fatores complexos e inter-relacionados que se originam de condiçôes biológicas, sociais e ambientais, expondo a mulher durante a gestação ${ }^{56}$.

Com base nos estudos analisados, foram notórios os agravos à saúde decorrentes do baixo peso/baixa reserva de adiposidade e do baixo peso ao nascer em crianças e adolescentes. Foi constatada a associação entre desnutrição e desenvolvimento intelectual abaixo da média, influenciada pela violência familiar. Pesquisas indicaram que as crianças com baixo peso ao nascer representam um risco aumentado para a hipertensão arterial, doença cardiovascular na vida adulta, anemia ferropriva, deficiência de vitamina $A$, transtornos cognitivo-comportamentais e de aprendizagem. Não obstante, fatores ocorridos na gestaçáo e refletidos nas medidas de tamanho ao nascer podem ocasionar problemas de saúde mental em etapas tardias.

Os estudos avaliados demonstraram que o baixo peso/baixa reserva de adiposidade ainda atinge um grande número de crianças e adolescentes, e que essa condição está relacionada a outras complicaçóes à saúde. Para superar a situação da desnutrição, medidas eficientes e urgentes são exigidas para o combate à pobreza e à fome, por meio da realização de políticas de inclusão social. Além disso, a atenção deve ser dada às condiçóes inadequadas de saneamento básico, aos baixos níveis de educação e aos serviços de saúde deficientes. 
Quadro 4: Estudos sobre a associação de baixo peso/baixa reserva de adiposidade com fatores de risco a saúde em crianças e adolescentes.

\begin{tabular}{|c|c|c|c|c|c|}
\hline Autor & Objetivo & $\begin{array}{c}\text { Faixa etária } \\
\text { (anos) }\end{array}$ & $\begin{array}{c}\text { Variáveis } \\
\text { antropométricas }\end{array}$ & $\begin{array}{l}\text { Desfechos } \\
\text { analisados }\end{array}$ & Principais achados \\
\hline Salgado et al. ${ }^{57}$ & $\begin{array}{c}\text { Avaliar as possíveis alteraçóes } \\
\text { na monitorizaçáo ambulato- } \\
\text { rial da PA em crianças com } \\
\text { baixo PN. }\end{array}$ & 8 a 11 & PN e IMC & PA & $\begin{array}{c}\text { As crianças com baixo PN } \\
\text { apresentaram PA mais } \\
\text { elevada e alteração do ritmo } \\
\text { circadiano da PA. }\end{array}$ \\
\hline Silva et al. ${ }^{13}$ & $\begin{array}{l}\text { Identificar os fatores de risco } \\
\text { associados à anemia ferropriva } \\
\text { em crianças. }\end{array}$ & 0 a 5 & $\mathrm{PN}$ & Anemia ferropriva & $\begin{array}{c}\text { Crianças que nasceram } \\
\text { com peso acima de } 3 \mathrm{~kg} \\
\text { apresentaram menor risco de } \\
\text { anemia. }\end{array}$ \\
\hline Riechi et al. ${ }^{58}$ & $\begin{array}{l}\text { Avaliar o impacto do nas- } \\
\text { cimento pré-termo e com } \\
\text { baixo peso no neurodesen- } \\
\text { volvimento, na cogniçáo e na } \\
\text { aprendizagem de crianças e } \\
\text { adolescentes. }\end{array}$ & 6 a 15 & $\mathrm{PN}$ & $\begin{array}{l}\text { Coordenação viso- } \\
\text {-motora, desenvol- } \\
\text { vimento psicomo- } \\
\text { tor geral, habilidade } \\
\text { viso-construtiva, } \\
\text { raciocínio mate- } \\
\text { mático, habilidade } \\
\text { tátil-cinestésica e } \\
\text { memória visual } \\
\end{array}$ & $\begin{array}{l}\text { Nascidos pré-termo e com } \\
\text { baixo peso ao nascer tiveram } \\
\text { resultados desfavoráveis para } \\
\text { a coordenação viso-motora, } \\
\text { desenvolvimento psicomotor } \\
\text { geral, habilidade viso-cons- } \\
\text { trutiva, raciocínio matemáti- } \\
\text { co, habilidade tátil-cinestési- } \\
\text { ca e memória visual. }\end{array}$ \\
\hline Gallo et al. ${ }^{59}$ & $\begin{array}{c}\text { Avaliar a associação entre } \\
\text { tamanho ao nascer e pro- } \\
\text { blemas de saúde mental aos } \\
11 \text { anos em uma coorte de } \\
\text { nascimentos. }\end{array}$ & $\begin{array}{c}\text { Média de } \\
11,3\end{array}$ & $\begin{array}{l}\text { Escores-z de peso, } \\
\text { comprimento, } \\
\text { perímetro cefálico e } \\
\text { IMC para idade }\end{array}$ & Saúde mental & $\begin{array}{c}\text { Crianças com déficit do } \\
\text { tamanho ao nascimento } \\
\text { apresentaram um maior ris- } \\
\text { co de desenvolver problemas } \\
\text { de saúde mental aos } 11 \text { anos. }\end{array}$ \\
\hline Souza et al. ${ }^{60}$ & $\begin{array}{l}\text { Avaliar o estado nutricional } \\
\text { antropométrico de crianças } \\
\text { e adolescentes com doença } \\
\text { falciforme. }\end{array}$ & 0 a 14 & $\begin{array}{l}\text { Peso para altura; } \\
\text { altura e IMC para } \\
\text { idade }\end{array}$ & Doença falciforme & $\begin{array}{l}\text { Crianças e adolescentes com } \\
\text { doença falciforme apresen- } \\
\text { taram maior prevalência de } \\
\text { baixa estatura e magreza em } \\
\text { relação à população geral. }\end{array}$ \\
\hline Silva et al. ${ }^{61}$ & $\begin{array}{c}\text { Verificar a direção e a mag- } \\
\text { nitude da associação entre } \\
\text { desnutrição e baixo desempe- } \\
\text { nho cognitivo. }\end{array}$ & 7 a 14 & IMC & $\begin{array}{l}\text { Desempenho } \\
\text { cognitivo }\end{array}$ & $\begin{array}{l}\text { Houve influência negativa } \\
\text { da desnutrição sobre desem- } \\
\text { penho cognitivo. }\end{array}$ \\
\hline $\begin{array}{l}\text { Siqueira; } \\
\text { Leandro }^{62}\end{array}$ & $\begin{array}{l}\text { Analisar estudos que associa- } \\
\text { ram o baixo peso ao nascer } \\
\text { e a proficiência motora em } \\
\text { crianças. }\end{array}$ & 1 a 10 & $\mathrm{PN}$ & Proficiência motora & $\begin{array}{l}\text { Crianças com PN abaixo de } \\
2.500 \mathrm{~g} \text { apresentaram algum } \\
\text { tipo de déficit motor. As } \\
\text { habilidades mais afetadas } \\
\text { foram as motoras finas e o } \\
\text { equilíbrio. } \\
\end{array}$ \\
\hline Ferreira et al. ${ }^{63}$ & $\begin{array}{c}\text { Identificar os fatores associa- } \\
\text { dos à hipovitaminose A em } \\
\text { crianças. }\end{array}$ & 0 a 5 & $\begin{array}{l}\text { PN e escores-z dos } \\
\text { índices de estatura, } \\
\text { peso e IMC para } \\
\text { idade }\end{array}$ & Hipovitaminose A & $\begin{array}{l}\text { O baixo PN foi associado } \\
\text { com hipovitaminose } \mathrm{A} \text {. }\end{array}$ \\
\hline
\end{tabular}

IMC: Índice de massa corporal; PN: Peso ao nascer; PA: Pressão arterial.

\section{Conclusão}

Os achados da presente revisão indicaram a coexistência de elevadas prevalências de obesidade/excesso de adiposidade e prevalências ainda preocupantes de baixo peso/baixa reserva de adiposidade entre crianças e adolescentes. A obesidade/excesso de adiposidade esteve associada aos indivíduos de sexo masculino, com menos irmãos, estudantes de escola particular e com nível socioeconômico médio ou alto, enquanto o baixo peso ao nascer se relaciona às crianças, principalmente na zona rural e com mãe de baixa escolaridade. Não obstante, pôde-se observar que ambos os extremos antropométricos estiveram associados a riscos à saúde. A obesidade/excesso de adiposidade associou-se à hipertensão, hiperglicemia, dislipidemia e síndrome metabólica, ao passo que o baixo peso/baixa reserva de adiposidade associou-se à anemia ferropriva, hipertensão, 
doença falciforme, hipovitaminose A, problemas de saúde mental, déficit motor, déficit cognitivo e mortalidade precoce.

Com base nesse contexto, açóes que visem diminuir a prevalência de extremos antropométricos são urgentes, especialmente para grupos em condiçóes sociodemográficas mais vulneráveis. Programas de segurança e reeducaçáo alimentar e de prática de atividade física relacionados à promoção da saúde, já nas séries iniciais do ensino fundamental, podem ser estratégias relevantes para enfrentar o problema.

\section{Referências}

1. Monteiro CA, Mondini L, Souza ALM, Popkin BM. Da desnutrição para a obesidade. A transição nutricional no Brasil. In: Monteiro CA. Velhos e novos males da saúde no Brasil: a evolução do país e de suas doenças. São Paulo: Hucitec; 1995. 248-55.

2. Pesquisa de Orçamentos Familiares 2008-2009. Rio de Janeiro: Instituto Brasileiro de Geografia e Estatística. 2010. Disponível em: http://www.ibge.gov.br/home/ estatistica/populacao/condicaodevida/pof/2008_2009/ POFpublicacao.pdf. [Acesso em 23 jan. 2011].

3. Rinald AEM, Nogueira PCK, Riyuzo MC, Olbrich-Neto J, Gabriel GFCP, Macedo CS, Burini RC. Prevalência de pressão arterial elevada em crianças e adolescentes do ensino fundamental. Rev Paul Pediatr. 2012;30(1):79-86.

4. Flores LS, Gaya AR, Petersen RDS, Gaya A. Tendência do baixo peso, sobrepeso e obesidade de crianças e adolescentes brasileiros. J Pediatr. 2013;89(5):456-61.

5. Ribeiro RQC, Lotufo PA, Lamounier JA, Oliveira RG, Soares JF, Botter DA. Fatores adicionais de risco cardiovascular associados ao excesso de peso em crianças e adolescentes. O Estudo do Coração de Belo Horizonte. Arq Bras Cardiol. 2006;86(6):408-18.

6. Victora CG, Adair L, Fall C, Hallal PC, Martorell R, Richter L, Sachdev HS. Maternal and child undernutrition: consequences for adult health and human capital. Lancet. 2008;26:340-57.

7. Kolotkin RL, Crosby RD, Kosloski KD, Williams GR. Development of a brief measure to assess quality of life in obesity. Obes Res. 2001;9(2):102-11.

8. Duquia RP, Dumith SC, Reichert FF, Madruga SW, Duro LN, Menezes AMB, Araújo CL. Epidemiologia das pregas cutâneas triciptal e subescapular elevadas em adolescentes. Cad Saúde Pública. 2008;24(1):113-21.

9. Mendonça MRT, Silva MAM, Rivera IR, Moura AA. Prevalência de sobrepeso e obesidade em crianças e adolescentes da cidade de Maceió. Rev Assoc Med Bras. 2010;56(2):192-6.

10. Benedet, J, Assis MAA, Calvo MCM, Andrade DF. Excesso de peso em adolescentes: explorando potenciais fatores de risco. Rev Paul Pediatr. 2013;31(2):172-81.
11. Tassitano RM, Barros MVG, Tenório MCM, Bezerra J, Hallal PC. Prevalência e fatores associados ao sobrepeso e à obesidade em adolescentes, estudantes de escolas de Ensino Médio de Pernambuco, Brasil. Cad Saúde Pública. 2009;25(12):2639-52.

12. Corso ACT, Caldeira GV, Fiates GMR, Schmitz BAS, Ricardo GD, Vasconcelos FAG. Fatores comportamentais associados ao sobrepeso e à obesidade em escolares do Estado de Santa Catarina. R Bras Est Pop. 2012;29(1):117-31.

13. Silva EB, Villani MS, Jahn AC, Cocco M. Fatores de risco associados a anemia ferropriva em crianças de 0 a 5 anos, em um município da Regiāo Noroeste do Rio Grande do Sul. Rev Min Enferm. 2011;15(2):165-73.

14. Minatto G, Pelegrini A, Silva DAS, Silva AF, Petroski EL. Composiçáo corporal inadequada em adolescentes: associação com fatores sociodemográficos. Rev Paul Pediatr. 2011;29(4):553-9.

15. Guedes DP, Neto JTM, Almeida MJ, Silva AJRM. Impacto de fatores sociodemográficos e comportamentais na prevalência de sobrepeso e obesidade de escolares. Rev Bras Cineantropom Desempenho Hum. 2010;12(4):221-31.

16. Mello ADM, Marcon SS, Hulsmeyer APCR, Cattai GBP, Ayres CSLS, Santana RG. Prevalência de sobrepeso e obesidade em crianças de seis a dez anos de escolas municipais de área urbana. Rev Paul Pediatr. 2010;28(1):48-54

17. Leal VS, Lira PIC, Oliveira JS, Menezes RCE, Sequeira LAS, Neto MAA, Andrade SLLS, Filho MB. Excesso de peso em crianças e adolescentes no Estado de Pernambuco, Brasil: prevalência e determinantes. Cad Saúde Pública. 2012;28(6):1175-82.

18. World Health Organization. Childhood overweight and obesity on the rise. 2010. Disponível em: http://www. who.int/dietphysicalactivity/childhood/en/. [Acesso em 20 ago. 2011].

19. Dietz WH. Periods of risk in childhood for the development of adult obesity - what do we need to learn? J Nutr. 1997;127(9):1884-6.

20. Zemel BS, Riley EM, Stallings VA. Evaluation of methodology for nutritional assessment in children: anthropometry, body composition and energy expenditure. Ann Rev Nutr. 1997;17:211-35.

21. Mcardle WD, Katch FI, Katch VL. Fisiologia do exercício: energia, nutrição e desempenho. Rio de Janeiro: Guanabara Koogan S. A.; 1998.

22. Misra A, Madhavan M, Vikram NK, Pandey RM, Dhingra V, Luthra K. Simple anthropometric measures identify fasting hyperinsulinemia and clustering of cardiovascular risk factors in Asian Indian adolescents. Metabolism. 2006;55(12):1569-73.

23. Montañes CE, Geraud AA, Sardiña NG, Bustos CL. Circunferencia de cintura, dislipidemia e hipertensión arterial en prepúberes de ambos sexos. An Pediatr (Barc). 2007;67(1):44-50. 
24. Chiara V, Sichieri R, Martins PD. Sensibilidade e especificidade de classificação de sobrepeso em adolescentes, Rio de Janeiro. Rev Saúde Pública. 2003;37(2):226-31.

25. Ronque ERV, Cyrino ES, Dórea VR, Júnior HS, Galdi EHG, Arruda M. Prevalência de sobrepeso e obesidade em escolares de alto nível socioeconômico em Londrina, Paraná, Brasil. Rev Nutr. 2005;18(6):709-17.

26. Ferrari TK, Ferrari GLM, Júnior JPS, Silva LJ, Oliveira LC, Matsudo, VKR. Modificaçóes da adiposidade em escolares de acordo com o estado nutricional: análise de 20 anos. J Pediatr. 2012;88(3):239-45.

27. Quadros TMB, Silva RCR, Gordia AP, Neto CSP. Excesso de peso em crianças: comparação entre o critério internacional e nacional de classificação do índice de massa corpórea. Rev Paul Pediatr. 2012;30(4):537-43.

28. Cesani MF, Garraza M, Sanchís MLB, Luis MA, Torres MF, Quintero FA, Oyhenart EE. Um estudo comparativo sobre o estado nutricional e corpo composição urbana e rural de alunos Brandsen District (Argentina). Plos One. 2013;8(1):1-7.

29. Vázquez-Nava F, Treviño-Garcia-Manzo N, VázquezRodríguez CF, Vázquez-Rodríguez EM. Association between family structure, maternal education level, and maternal employment with sedentary lifestyle in primary school-age children. J Pediatr. 2013;89(2):145-50.

30. Minatto G, Nascimento TBR, Ribeiro RR, Santos KD, Petroski EL. A associação entre a adiposidade corporal e a aptidão musculoesquelética em meninos é mediada pelo nível econômico? Rev Bras Cineantropom Desempenho Hum. 2014;16(1):116-28.

31. Campbell I. The obesity epidemic: can we turn the tide? Heart. 2003;89(2):22-4.

32. Secretaria de Educação Fundamental. Parâmetros curriculares nacionais: introdução aos parâmetros curriculares nacionais. Brasília: MEC/SEF;1997.

33. Guedes PD. Educação para saúde mediante programas de educação física escolar. Motriz: Rev Educ Fis. 1999;5(1):10-4.

34. Mazzoccante RP, Sousa IC, Sales MM, Melo GF, Neto WB, Campbell CSG. A avaliação antropométrica em crianças tem sido realizada nas escolas para a prevençáo do sobrepeso e obesidade? Rev Mac Educ Fís Esp. 2013;12(2):76-8.

35. Freedman DS, Mei Z, Srinivasan SR, Berenson GS, Dietz WH. Cardiovascular Risk Factors and Excess Adiposity Among Overweight Children and Adolescents: The Bogalusa Heart Study. J Pediatr. 2007;150(1):12-7.

36. Ferreira JS, Aydos RD. Adiposidade corporal e hipertensão arterial em crianças e adolescentes obesos. Rev Bras Prom Saúde. 2009;22(2):88-93.

37. Martins RV, Campos W, Bozza R, Filho VCB, Silva MP. Prevalência de hipertensão arterial e sua associação com sobrepeso e obesidade: estudo de base escolar. Rev Bras Cineantropom Desempenho Hum. 2013;15(5):551-60.

38. Reuter CP, Burgos LT, Camargo MD, Possuelo LG, Reckziegel MB, Reuter EM, Meinhardt FP, Burgos MS.
Prevalence of obesity and cardiovascular risk among children and adolescents in the municipality of Santa Cruz do Sul, Rio Grande do Sul. São Paulo Med J. 2013;131(5):323-30.

39. Silva LS, Madrid B, Martins CM, Queiroz JL, Dutra MT, Silva FM. Influência de fatores antropométricos e atividade física na pressão arterial de adolescentes de Taguatinga, Distrito Federal, Brasil. Motri. 2013;9(1);13-22.

40. Sousa MACA, Guimarães ICB, Daltro C, Guimarães AC. Associação entre peso de nascimento e fatores de risco cardiovascular em adolescentes. Arq Bras de Cardiol. 2013;101(1):9-17.

41. Lacerda EMA, Accioly E, Faria IG, Costa VM. Práticas de Nutrição Pediátrica. São Paulo: Editora Atheneu;2006.

42. World Health Organization. Recommended definitions, terminology and format for statistical tables related to the perinatal period and use of a new certificate for cause of perinatal deaths. Modifications recommended by FIGO as amended October 14, 1976. Acta Obstet Gynecol Scand. 1977;56:247-53.

43. Monteiro CA, Conde WL, Castro IRR. A tendência cambiante da relação entre escolaridade e risco de obesidade no Brasil (1975-1997). Cad Saúde Pública. 2003;19(1):67-75.

44. Victora CG, Vaughan JP, Kirkwood BR, Martines JC, Barcelos LB. Risk factors for malnutrition in Brazilian children: the role of social and environmental variable. Bulletin of the World Health Organization. 1986;64(2):299-309.

45. Pelegrini A, Silva DAS, Petroski EL, Glaner MF. Estado nutricional e fatores associados em escolares domiciliados na área rural e urbana. Rev Nutr. 2010;23(5):839-46.

46. Silveira KBR, Alves JFR, Ferreira HS, Sawaya AL, Florêncio TMMT. Associação entre desnutrição em crianças moradoras de favelas, estado nutricional materno e fatores socioambientais. J Pediatr. 2010;86(3):215-20.

47. Ferreira HS, Luciano SCM. Prevalência de extremos antropométricos em crianças do estado de Alagoas. Rev Saúde Pública. 2010;44(2):377-80.

48. Krinski K, Elsangedy HM, Hora S, Rech CR, Legnani E, Santos BV, Campos W, Silva SG. Estado nutricional e associação do excesso de peso com gênero e idade de crianças e adolescentes. Rev Bras Cineantropom Desempenho Hum. 2011;13(1):29-35.

49. Barros MS, Fonseca VM, Meio MDBB, Chaves CR. Excesso de peso entre adolescentes em zona rural e a alimentação escolar oferecida. Cad Saúde Colet. 2013;21(2):201-8.

50. Benício MHD, Martins APB, Venancio SI, Barros AJD. Estimativas da prevalência de desnutrição infantil nos municípios brasileiros em 2006. Rev Saúde Pública. 2013;47(3):560-70.

51. Guedes DP, Almeida FN, Neto JTM, Maia MFM, Tolentino TM. Baixo peso corporal/magreza, sobrepeso e obesidade de crianças e adolescentes de uma região brasileira de baixo desenvolvimento econômico. Rev Paul Pediatr. 2013;31(4):437-43. 
52. Pinho L, Flávio EF, Santos SHS, Botelho ACC, Caldeira, AP. Excesso de peso e consumo alimentar em adolescentes de escolas públicas no norte de Minas Gerais, Brasil. Cad Saúde Colet. 2014;19(1):67-74.

53. Correia LL, Silva AC, Campos JS, Andrade FMO, Machado MMT, Lindsay AC, Leite AJM, Rocha HAL, Cunha AJLA. Prevalence and determinants of child undernutrition and stunting in semiarid region of Brazil. Rev Saúde Pública. 2014;48(1):19-28.

54. Recine E, Radaelli P. Obesidade e Desnutrição. Brasília NUT/FS/UnB - ATAN/DAB/SPS/MS. Disponível em: http://bvsms.saude.gov.br/bvs/publicacoes/obesidade_desnutricao.pdf. [Acesso em 28 fev. 2011].

55. Sawaya AL. Desnutrição: consequências em longo prazo e efeitos da recuperação nutricional. Est Av. 2006;20(58):147-58.

56. Lima GSP, Sampaio HAC. Influência de fatores obstétricos, socioeconômicos e nutricionais da gestante sobre o peso do recém-nascido: estudo realizado em uma maternidade em Teresina, Piauí. Rev Bras Saude Mater Infant. 2004;4(3):253-61.

57. Salgado CM, Jardim PCBV, Teles FBG, Nunes MC. Baixo peso ao nascer como marcador de alteraçóes na monitorização ambulatorial da pressão arterial. Arq Bras Cardiol. 2009;92(2):113-21.

58. Riechi TIJ, Moura-Ribeiro MVL, Ciasca SM. Impacto do nascimento pré-termo e com baixo peso na cognição, comportamento e aprendizagem de escolares. Rev Paul Pediatr. 2011;29(4):495-501.

59. Gallo EAG, Anselmi L, Dumith SC, Scazufca M, Menezes AMB, Hallal PC, Matijasevich A. Tamanho ao nascer e problemas de saúde mental aos 11 anos em uma coorte brasileira de nascimentos. Cad Saúde Pública. 2011;27(8):1622-32.

60. Souza KCM, Araújo PIC, Souza-Junior PRB, Lacerda EMA. Baixa estatura e magreza em crianças e adolescentes com doença falciforme. Rev Nutr. 2011;24(6):853-62.

61. Silva RCR, Assis AMO, Hasselmann MH, Santos LM, Pinto EJ, Rodrigues LC. Influência da violência familiar na associação entre desnutriçáo e baixo desenvolvimento cognitivo. J Pediatr. 2012;88(2):149-54.

62. Siqueira AKM, Leandro CG. Baixo peso ao nascer e proficiência motora em crianças: uma revisão sistemática. Rev Nutr. 2012;25(6):775-84.

63. Ferreira HS, Moura RMM, Assunção ML, Horta BL. Fatores associados à hipovitaminose $\mathrm{A}$ em crianças menores de cinco anos. Rev Bras Saude Mater Infant. 2013;13(3):223-35. 